\title{
Magnetic solid-phase extraction of heterocyclic pesticides in environmental water samples using metal-organic frameworks coupled to high performance liquid chromatography determination
}

\author{
Jiping Ma ${ }^{\mathrm{a}, *}$, Gege $\mathrm{Wu}^{\mathrm{a}}$, Shuang $\mathrm{Li}^{\mathrm{a}}$, Weiqiang Tan ${ }^{\mathrm{a}}$, Xiaoyan Wang ${ }^{\mathrm{b}, \mathrm{c}}$, Jinhua $\mathrm{Li}^{\mathrm{b}}$, \\ Lingxin Chen ${ }^{\mathrm{b}, *}$ \\ a School of Environmental \& Municipal Engineering, State-Local Joint Engineering Research Center of Urban Sewage Treatment and Resource Recovery, \\ Qingdao University of Technology, Qingdao 266033, China \\ ${ }^{\mathrm{b}}$ Key Laboratory of Coastal Environmental Processes and Ecological Remediation, Research Centre for Coastal Environmental Engineering and Technology, \\ Yantai Institute of Coastal Zone Research, Chinese Academy of Sciences, Yantai 264003, China \\ ' School of Pharmacy, Binzhou Medical University, Yantai 264003, China
}

\section{A R T I C L E I N F O}

\section{Article history:}

Received 26 January 2018

Received in revised form 9 April 2018

Accepted 14 April 2018

Available online 16 April 2018

\section{Keywords:}

Heterocyclic pesticide

Magnetic solid-phase extraction

Metal-organic framework

Water sample

\begin{abstract}
A B S T R A C T
A simple method of magnetic solid-phase extraction (MSPE) coupled to high performance liquid chromatography (HPLC) was developed for the simultaneous extraction and determination of four kinds of heterocyclic pesticides (carbendazim, triadimefon, chlorfenapyr and fenpyroximate) in environmental water samples. Magnetic metal-organic frameworks (MOFs) of type MOF-5 were prepared and used as adsorbents of MSPE. Several main parameters influencing MSPE efficiency were investigated, including amount of magnetic MOF-5, sample solution pH, extraction time, salt concentration, type and volume of desorption solvents and desorption time. Under optimal conditions, the MSPE-HPLC method presented fast simple separation and analysis, and excellent linearity in the range of $0.3-500.0 \mu \mathrm{g} / \mathrm{L} \mathrm{for}$ carbendazim and triadimefon, and $0.1-500.0 \mu \mathrm{g} / \mathrm{L}$ for chlorfenapyr and fenpyroximate, with correlation coefficients $(r)$ higher than 0.9992 . High sensitivity with limits of detection and quantification ranging from $0.04-0.11 \mu \mathrm{g} / \mathrm{L}$ and $0.13-0.35 \mu \mathrm{g} / \mathrm{L}$, respectively, were achieved, as well as good precision with relative standard deviations of 2.98-7.11\% (intra-day) and 3.31-7.12\% (inter-day). Furthermore, the method was successfully applied to reservoir and Yellow River water samples, and satisfactory recoveries at three spiked concentration levels were between $80.20 \%$ and 108.33\%.The magnetic MOF-5 composites based MSPE followed by HPLC proved promising for convenient and efficient determination of heterocyclic pesticides in environmental water samples.
\end{abstract}

(c) 2018 Elsevier B.V. All rights reserved.

\section{Introduction}

Heterocyclic pesticides as a large class of pesticides are mainly used to control insects and pathogens for a variety of rice, vegetable, fruit trees, decorative plants and so on. But meanwhile, for those non-target organisms, these heterocyclic pesticides also have exhibited high toxicity or harmful side effect [1]. For example, chlorfenapyr, a kind of widely used heterocyclic pesticides, is very toxic to aquatic organisms. They can persist for a long time in the environment because the heterocyclic rings of these compounds are difficult to be broken and their biodegradability is slow. Due to extensive and inappropriate use, their residues have been

\footnotetext{
* Corresponding authors.

E-mail addresses: majiping2012@163.com (J. Ma), lxchen@yic.ac.cn (L. Chen).
}

observed in vegetables, agricultural products and environmental soils or waters [2,3]. The residues of heterocyclic pesticides in environmental water may cause long-term adverse effects to the aquatic environment, and even some heterocyclic pesticides may do harm to human health. Therefore, it is extremely essential to develop convenient, sensitive and rapid methods for heterocyclic pesticides determination in environmental waters.

Besides traditionally available chromatographic technologies for separation and determination of heterocyclic pesticides [4-8], sample pretreatment procedures are crucial for the whole analysis process, which aim at enriching targeted analytes and eliminating matrix effects from complex samples. Some pretreatment techniques for the extraction of heterocyclic pesticides from water samples have been reported, such as solid-phase extraction (SPE) $[9,10]$, solid-phase microextraction (SPME) [11], and dispersive liquid-liquid microextraction (DLLME) [12,13]. Amongst them, SPE 
technique can provide high enrichment factor to obtain lower detection limit, and has been widely used for preconcentration of trace analytes in water samples. For instance, our group has used multi-walled carbon nanotubes (MWCNTs) as SPE adsorbents combined with HPLC-DAD for enrichment and determination of pyrazole/pyrrole pesticides in water [14]. However, SPE method still has some problems, such as, the SPE columns are likely to be blocked by complicated water samples, and the extraction process is time-consuming.

As a deformation mode of SPE, magnetic SPE (MSPE) technique nowadays has attracted much interest and have been increasingly utilized in sample pretreatment of environmental water [15,16]. In MSPE mode, a small amount of magnetic adsorbents can be well dispersed in sample solution to trap target analytes and be rapidly collected under magnetic fields. Compared with traditional SPE, MSPE is time saving, and magnetic adsorbents can be easily separated from water solution without employing complicated devices $[15,16]$. In MSPE, it is the key to prepare and select magnetic adsorbents for different compounds to obtain high enrichment efficiency.

Metal organic frameworks (MOFs) are new functional materials with various transition metal ions interconnected by a variety of organic ligands. MOFs have some excellent characteristics such as enormous surface area, ultrahigh porosity, and tuneable pore size, and they have demonstrated great application potentials in gas storage [17-19], drug delivery [20,21], heterogeneous catalysis applications [22,23], and sample pretreatment [24,25]. As for sample pretreatment, MOFs have often been used as adsorbents of SPE [24-28] and SPME $[24,25,29,30]$. However, not all MOFs can be used in environmental water samples, because some MOFs have limitations in liquid phase adsorption due to their relatively low stability in water and hydrophilic nature [31,32]. Recently, several magnetic MOFs as MSPE adsorbents for water analysis have been reported, and they exhibit good dispersibility in water together with good adsorption efficiencies. For example, magnetic MIL-101 has been used for MSPE of polycyclic aromatic hydrocarbons (PAHs) [33] and pyrazole/pyrrole pesticides [34] in environmental water samples. Magnetic HKUST-1 based MSPE has been used in determination of palladium in environmental samples [35], as well as $\mathrm{Fe}_{3} \mathrm{O}_{4} @ \mathrm{ZIF}-8$ microspheres for MSPE enrichment of phthalate esters [36]. The magnetic MOFs materials have been demonstrated to be stable in water samples. However, the metal center $(\mathrm{Cr})$ of this material is toxic. MOF-5 (Zn) material synthesized by solvothermal method has been proved to be water stable and eco-friendly. For instance, MOF-5 has been utilized for SPE of thiols from wastewater [37]. Also, magnetic MOF-5 has been used for MSPE. For example, MOF$5-\mathrm{Fe}_{3} \mathrm{O}_{4}$ nanoparticles have been synthesized for the enrichment of colchicine in root of colchicium extracts and plasma samples [38]. Amino functionalized $\mathrm{Fe}_{3} \mathrm{O}_{4}$ nanoparticles have been combined with MOF-5 and then presented effective MSPE of PAHs and gibberellic acids from environmental, food and plant samples [39]. However, in those reports magnetic MOF-5 is not used in environmental water samples.

Taking account of the advantages of MOF materials and MSPE procedures, in this study, we prepared magnetic MOF-5 composites and used them for MSPE of four widely used heterocyclic pesticides, namely carbendazim, triadimefon, chlorfenapyr and fenpyroximate, in environmental water samples. Several main factors that possibly influenced the MSPE efficiency were systematically optimized. Then the developed MSPE-HPLC method was validated and applied to real water samples.

\section{Experimental}

\subsection{Chemical reagents and solutions}

Ferric chloride hexahydrate $\left(\mathrm{FeCl}_{3} \cdot 6 \mathrm{H}_{2} \mathrm{O}\right), \mathrm{Zn}\left(\mathrm{NO}_{3}\right)_{2} \cdot 6 \mathrm{H}_{2} \mathrm{O}$, hydrochloricacid $(\mathrm{HCl})$ and terephthalic acid were purchased from Sinopharm Chemical Reagent Co. Ltd. (Shanghai, China). $N, N^{\prime}$-Dimethylformamide (DMF) and glycol were purchased from Fuyu Fine Chemical Co. Ltd. (Tianjin, China). Sodium acetate was obtained from Aibi (Shanghai, China). Cholamine was supplied by Sanaisi (Shanghai, China). All organic solvents are of HPLC grade. Methanol, acetonitrile and acetone were purchased from TEDIA (USA). Ethyl acetate was obtained from Tianjin Guangfu Fine<smiles>COC(=O)Nc1nc2ccccc2[nH]1</smiles>

Carbendazim

kow $\log \mathrm{P}=1.51$ $\mathrm{pKa}=5.0$

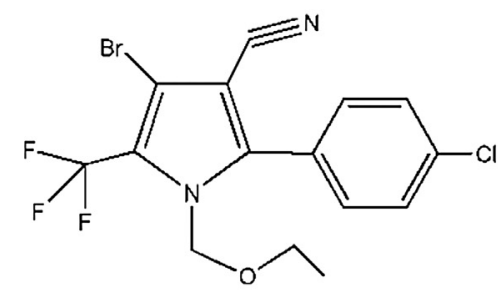

Chlorfenapyr<smiles>CC(C)(C)C(=O)C(Oc1ccc(Cl)cc1)N1CN=CN1</smiles>

Triadimefon

kow $\log P=3.11$

kow $\log \mathrm{P}=4.50$<smiles>Cn1ncc(/C=N/OCc2ccc(C(=O)OC(C)(C)C)cc2)c1Oc1ccccc1</smiles>

Fenpyroximate

kow $\log \mathrm{P}=5.96$

Fig. 1. Structural formulas of the four analyzed heterocyclic pesticides. 
Chemicals Institute (China). All chemicals were of at least analytical grade. Ultrapure water $(18.2 \mathrm{M} \Omega \mathrm{cm})$ was used throughout the present study for aqueous solution preparation, which was obtained from a model D-24UV ultra-pure water system (Millipore, USA).

Analytical standards of the four heterocyclic pesticides (carbendazim, triadimefon, chlorfenapyr and fenpyroximate) were purchased from the Testing Center of the Shanghai Pesticide Research Institute (Shanghai, China), and Fig. 1 shows their structural formulas. A stock solution of each analyte at $1000 \mathrm{mg} / \mathrm{L}$ was prepared by dissolving solid pesticides standards in methanol, and stored at $-18^{\circ} \mathrm{C}$ in the dark.

Real water samples were collected from the Qingdao Qianhan Reservoir, Nuocheng Reservoir and the Yellow River. All the water samples were filtered through $0.45 \mu \mathrm{m}$ membrane (Tianjin Jinteng Experiment Equipment Ltd, Co., Tianjin, China) and stored in brown glass bottles at $4{ }^{\circ} \mathrm{C}$ prior to analysis.

\subsection{Apparatus}

Four heterocyclic pesticides were analyzed using an Agilent 1100 liquid chromatographic system coupled with DAD and FLD detector. HPLC separation was performed using a ZORBAXSB- $\mathrm{C}_{18}$ column $(100 \times 4.6 \mathrm{~mm}, 5 \mu \mathrm{m})$ at room temperature. The sample injection volume was $20 \mu \mathrm{L}$. The FLD excitation/emission wavelengths were selected at $270 / 315 \mathrm{~nm}$ for carbendazim, and DAD absorbance was monitored at $215 \mathrm{~nm}$ for triadimefon, chlorfenapyr, and fenpyroximate. The mobile phase was a mixture of methanol and water. Gradient elution program used was as fol- lows: $0 \mathrm{~min}$, isocratic $60 \%$ methanol, the flow rate was $0.7 \mathrm{~mL} / \mathrm{min}$; $4 \mathrm{~min}$, isocratic $65 \%$ methanol, the flow rate was $0.7 \mathrm{~mL} / \mathrm{min} ; 8 \mathrm{~min}$, isocratic $73 \%$ methanol, the flow rate was $1 \mathrm{~mL} / \mathrm{min} ; 13-18 \mathrm{~min}$, isocratic $80 \%$ methanol, the flow rate was $1 \mathrm{~mL} / \mathrm{min}$. Under these optimum HPLC conditions by our investigations, four pesticides can be well separated from each other.

A Fourier-transform infrared spectrometry (FT-IR, Frontier, Perkin Elmer, USA), and an SUPRA 55 scanning electron microscope (ZEISS, Germany) were used to characterize the synthesized magnetic MOF-5 composites. The value of saturation magnetization was studied by VSM 7307 vibrating sample magnetometer (VSM) (Lake Shore, USA) with an applied field between $-10,000$ and 10,000 $\mathrm{O}_{\mathrm{e}}$ at room temperature. The zeta potential change and the dynamic light scattering (DLS) of magnetic MOF-5 were measured by ZetaPlus (Brookhavaen, USA). The X-ray diffractometer (XRD) patterns were recordedon D8 Advance (Bruker, USA).

\subsection{Preparation of magnetic MOF-5}

The magnetic MOF-5 was prepared using a solvothermal method. Firstly, the amino functionalized $\mathrm{Fe}_{3} \mathrm{O}_{4}$ nanoparticles $\left(\mathrm{NH}_{2}-\mathrm{Fe}_{3} \mathrm{O}_{4}\right)$ were synthesized by a hydrothermal method [40]; then, the $\mathrm{NH}_{2}-\mathrm{Fe}_{3} \mathrm{O}_{4}$ nanoparticles were modified with MOF-5 using a solvothermal method [39]. The preparation process was illustrated in Fig. 2. In short, terephthalic acid and $\mathrm{Zn}\left(\mathrm{NO}_{3}\right)_{2} \cdot 6 \mathrm{H}_{2} \mathrm{O}$ was dissolved in DMF, then $\mathrm{NH}_{2}-\mathrm{Fe}_{3} \mathrm{O}_{4}$ was uniformly dispersed into the above mixed solution. The obtained mixture was transferred into the autoclave, which was sealed and heated. Then, the

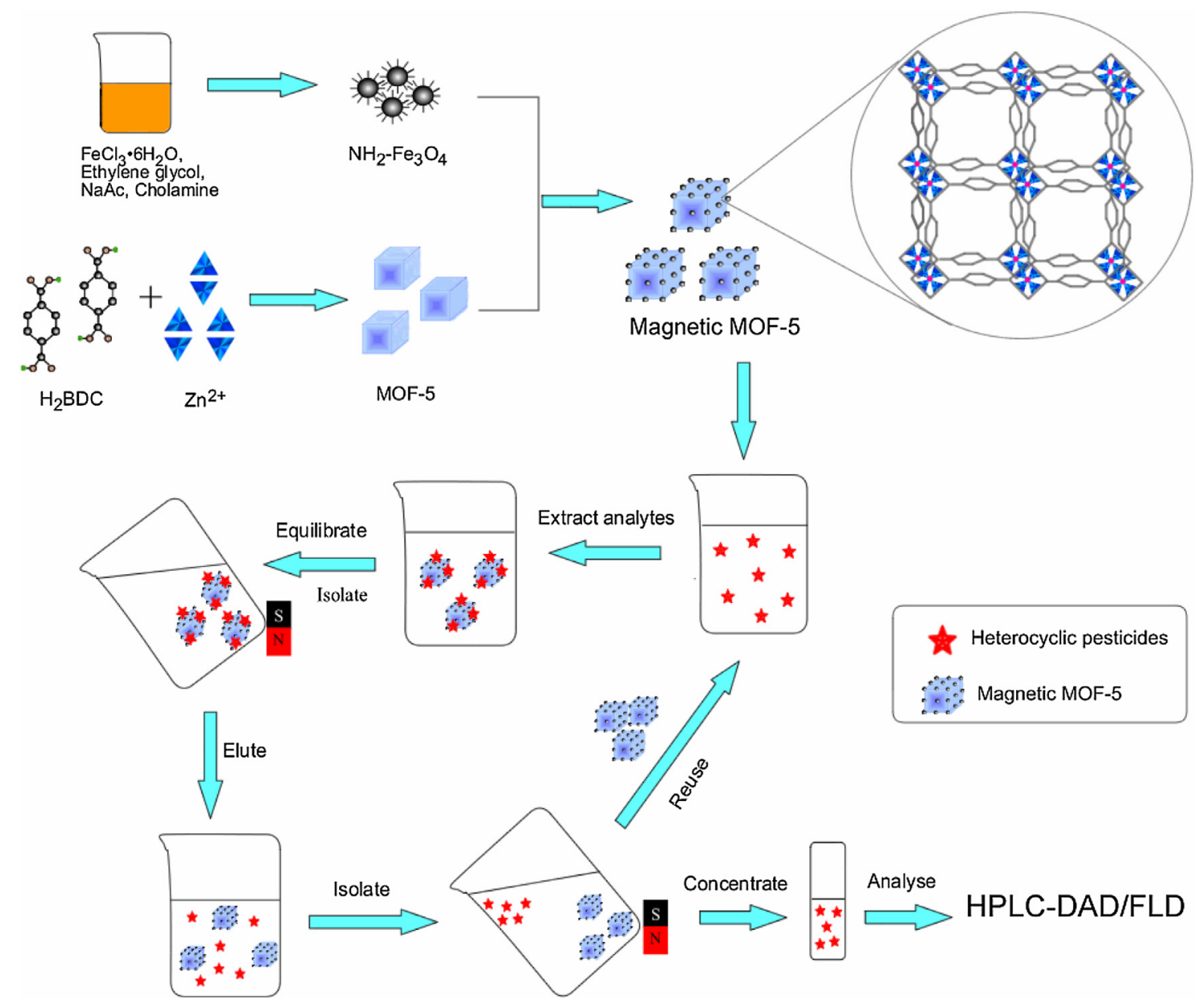

Fig. 2. Schematic illustration of the preparation process for the magnetic MOF-5 and the MSPE procedure. 
magnetic particles were collected by a magnet and washed several times with solvent. The product was dried under vacuum.

\subsection{MSPE procedure}

Before the MSPE procedure, $7 \mathrm{mg}$ of magnetic MOF- 5 composites were placed into a $100 \mathrm{~mL}$ beaker, and then $60 \mathrm{~mL}$ of water sample was added into the beaker. The magnetic MOF-5 composites were dispersed in the mixture solution under ultrasonication for $12 \mathrm{~min}$ for extraction of pesticides. The supernatant water was decanted and discarded; magnetic MOF-5 composites were gathered by attaching an external magnet to the outside bottom of the beaker, and the sedimentation time was about $4 \mathrm{~min}$, ensuring that the majority of absorbents were collected. Wet magnetic MOF-5 composites were then eluted twice by $3 \mathrm{~mL}$ of ethyl acetate each time, and the elution procedure was twice conducted, resulting in that the used total volume of ethyl acetate was $6 \mathrm{~mL}(2 \times 3 \mathrm{~mL})$. All gathered ethyl acetate extracts were transferred into a vial and evaporated at room temperature with a gentle nitrogen stream to approximately dry, then diluted to $0.2 \mathrm{~mL}$ with methanol, which was filtered with $0.45 \mu \mathrm{m}$ nylon membrane prior to HPLC analysis. After one MSPE process, in order to reuse the same adsorbent for new extraction of the four heterocyclic pesticides, the magnetic MOF-5 composites were washed with ethyl acetate solution for 3 times, and then the composite adsorbent was dried at $75^{\circ} \mathrm{C}$ for new extraction. The magnetic MOF- 5 based MSPE procedure was schematically illustrated in Fig. 2.

\section{Results and discussion}

\subsection{Characterization of the magnetic MOF-5 composites}

The prepared magnetic MOF-5 composites were characterized by SEM, VSM, FT-IR and XRD. Fig. S1a shows the morphological structure of magnetic MOF- 5 composites observed by SEM. As seen, the external surfaces of cubical MOF- 5 crystals were decorated with $\mathrm{Fe}_{3} \mathrm{O}_{4}$ nanoparticles, which demonstrated the successful assembly of MOF-5 crystals with $\mathrm{NH}_{2}-\mathrm{Fe}_{3} \mathrm{O}_{4}$ nanoparticles. As shown from the DLS curves in Fig. S2, the average diameter of magnetic MOF5 composites was about $10 \mu \mathrm{m}$ (Fig. S2a), much larger than that of $\mathrm{NH}_{2}-\mathrm{Fe}_{3} \mathrm{O}_{4}$ (about $80 \mathrm{~nm}$, Fig. S1b and 2b). The polydispersity index (PDI) of magnetic MOF-5 composites was obtained of 0.568 according to the DLS measurement. According to related researches such as [40], mentioning that PDI below 0.5 suggested a monodisperse system, ranging from 0.5 to 0.7 suggested an approximate monodisperse system, above 0.7 suggested not a monodisperse system, our synthesized magnetic MOF-5 composites were approximately monodisperse.

Fig. S3 shows the VSM curve of magnetic MOF-5 composites. As seen, the saturation magnetization value of magnetic MOF-5 was $32 \mathrm{emu}^{-1}$, which was sufficient for magnetic separation by means of an external magnetic field. And the magnetic MOF-5 composites were found to be well dispersed in water and isolated from the aqueous solution by a magnet (inset of Fig. S3). So, the composites had good paramagnetic characteristics.
Fig. S4 shows the FT-IR spectra of $\mathrm{NH}_{2}-\mathrm{Fe}_{3} \mathrm{O}_{4}$ (a), and magnetic MOF-5 composites (b) to examine the chemical structures. In Fig. S4a, the absorption peak at $580 \mathrm{~cm}^{-1}$ might be attributed to the Fe-O-Fe vibration; the absorption peaks at $3385 \mathrm{~cm}^{-1}$ and $1610 \mathrm{~cm}^{-1}$ could be ascribed to $\mathrm{N}-\mathrm{H}$ vibration, respectively. In Fig. $\mathrm{S} 4 \mathrm{~b}$, the absorption peak at $3400 \mathrm{~cm}^{-1}$ was possibly from $\mathrm{O}-\mathrm{H}$ stretching vibration. The peak at $1750 \mathrm{~cm}^{-1}$ could be ascribed to stretching vibration of $\mathrm{C}=\mathrm{O}$ from the terephthalic acid. The peak at $1580 \mathrm{~cm}^{-1}$ very likely belonged to $\mathrm{C}=\mathrm{C}$ of benzene ring. The absorption peak at $589 \mathrm{~cm}^{-1}$ might be attributed to the $\mathrm{Fe}-\mathrm{O}-\mathrm{Fe}$ vibration. All the characteristic peaks indicated that the magnetic MOF-5 composites were attained. The results were consistent with that reported patterns of magnetic MOF-5 [39].

Fig. S5 shows the XRD patterns of $\mathrm{NH}_{2}-\mathrm{Fe}_{3} \mathrm{O}_{4}(\mathrm{a})$, and magnetic MOF-5 composites (b). As seen, characteristic peaks in Fig. S5a at $2 \theta=30.1^{\circ}, 35.5^{\circ}, 43.1^{\circ}, 57.0^{\circ}$ and $62.7^{\circ}$, corresponding to the (220), (311), (400), (511) and (440) planes can be attributed to the crystalline structure of $\mathrm{NH}_{2}-\mathrm{Fe}_{3} \mathrm{O}_{4}$, which is in accordance with that reported [41]. As shown in Fig.S5b, The two main diffraction peaks at $2 \theta=6.9^{\circ}(\langle 200\rangle$ plane, $d=12.9 \AA)$ and $9.6^{\circ}(\langle 220\rangle$ plane, $d=9.2 \AA)$ indicated the cubic lattice of the MOF-5 [37], and the observed pecks at $2 \theta=13.7^{\circ}, 15.4^{\circ}, 20.7^{\circ}$ and $25.3^{\circ}$ are also in good agreement with that reported XRD patterns of MOF-5 [38].

Additionally, in order to check the stability of magnetic MOF-5 composites after ultrasonation, both FT-IR and XRD characterizations of the composites before (curve a) and after (curve b) ultrasonication were carried out, as shown in Fig. S6. The FT-IR absorption peaks (Fig. S6A) at 1750 and $1580 \mathrm{~cm}^{-1}$ (ascribed to stretching vibration of $\mathrm{C}=\mathrm{O}$ and $\mathrm{C}=\mathrm{C}$ of benzene ring from the terephthalic acid) and the absorption peaks at $589 \mathrm{~cm}^{-1}$ (related to the $\mathrm{Fe}-\mathrm{O}-\mathrm{Fe}$ vibration) can also be observed after ultrasonication. The XRD patterns comparison of magnetic MOF-5 after and before ultrasonication in Fig. S6 B clearly suggested that the crystalline structures of magnetic MOF-5 did not collapse. These results indicated that the chemical structures of magnetic MOF-5 did not change after ultrasonication.

\subsection{Adsorption capacity of the magnetic MOF-5 composites}

The adsorption isotherms of four pesticides onto the prepared magnetic MOF-5 adsorbents were determined by batch experiments. A series of solutions with different initial pesticides concentrations ranging from 5 to $40 \mathrm{mg} \mathrm{L}^{-1}(\mathrm{pH}=6.0)$ with $0.1 \mathrm{mg} / \mathrm{mL}$ of magnetic MOF-5 were placed to a shaker for $12 \mathrm{~min}$ at $25^{\circ} \mathrm{C}$. The adsorption capacity $q_{\mathrm{e}}\left(\mathrm{mg} \mathrm{g}^{-1}\right)$ was calculated based on Eq. (1):

$q_{\mathrm{e}}=\frac{\left(C_{0}-C_{\mathrm{e}}\right) V}{W}$

Here, $C_{0}$ and $C_{\mathrm{e}}$ mean the initial and the equilibrium concentrations of pesticides in solutions $\left(\mathrm{mg} \mathrm{L}^{-1}\right) ; W$ is the mass of the adsorbent $(\mathrm{g})$, and $V$ is the volume of the solution (L). As shown in Fig. S7, the maximum capacities of the magnetic MOF-5 materials were $84 \mathrm{mg} \mathrm{g}^{-1}$ for carbendazim, $81 \mathrm{mg} \mathrm{g}^{-1}$ for triadimefon, $181 \mathrm{mg} \mathrm{g}^{-1}$ for chlorfenapyr and $139 \mathrm{mg} \mathrm{g}^{-1}$ for fenpyroximate. As far as we know, the adsorption capacities simultaneously for the four pesticides by other adsorbents have not been reported yet.

Table 1

Related analytical parameters of the magnetic MOF-MSPE-HPLC method for determination of four heterocyclic pesticides.

\begin{tabular}{|c|c|c|c|c|c|}
\hline Heterocyclic pesticides & Regression equation $^{\mathrm{a}}$ & Correlation coefficient $(r)$ & Linearrange $(\mu \mathrm{g} / \mathrm{L})$ & $\operatorname{LOD}(\mu \mathrm{g} / \mathrm{L})$ & $\operatorname{LOQ}(\mu \mathrm{g} / \mathrm{L})$ \\
\hline Carbendazim & $y=0.8901 x+0.1952$ & 0.9990 & $0.3-500$ & 0.10 & 0.32 \\
\hline Triadimefon & $y=1.0322 x+0.0505$ & 0.9984 & $0.3-500$ & 0.11 & 0.35 \\
\hline Chlorfenapyr & $y=1.8762 x+0.1773$ & 0.9998 & $0.1-500$ & 0.04 & 0.13 \\
\hline Fenpyroximate & $y=2.3556 x+1.9644$ & 0.9998 & $0.1-500$ & 0.04 & 0.14 \\
\hline
\end{tabular}

a $x=$ concentration $(\mu \mathrm{g} / \mathrm{L}), y=$ area. 
For comparison, we carried out related experiments using the our reported MWCNTs [14] as the adsorbents. The adsorption capacity for fenpyroximate was obtained of $320 \mathrm{mg} \mathrm{g}^{-1}$, while the other three pesticides (carbendazim, triadimefon, chlorfenapyr) couldn't be absorbed by the MWCNTs. Hence, we can say that the magnetic MOF-5 had high adsorption capacities for the targeted analytes, which would be in favor of MSPE.

\subsection{Optimization of MSPE conditions}

Several major experimental parameters that could affect the MSPE performance were investigated, including amount of magnetic MOF-5, sample solution $\mathrm{pH}$, extraction time, type and volume of desorption solvents, salt concentration, and elution time. $250 \mu \mathrm{g} / \mathrm{L}$ of spiked ultrapure water sample was used under different experimental conditions, and the optimization was conducted by three parallel experiments.

\subsubsection{Effect of amount of magnetic MOF-5 composites}

Insufficient amount of adsorbent would cause the breakthrough of the analytes whereas higher amount will increase the cost of the analytical procedure. Herein, 4-10 mg of magnetic MOF-5 was used to investigate the adsorbent effect on MSPE efficiency of four pesticides. As shown in Fig. $3 \mathrm{~A}$, the extraction efficiency increased when the amount of magnetic MOF-5 composites increased from 4 to $7 \mathrm{mg}$. Then, the extraction efficiencies of carbendazim and triadimefon did not change much when the amount of magnetic MOF-5 increased from 7 to $10 \mathrm{mg}$, while the extraction efficiencies of chlorfenapyr and fenpyroximate slightly decreased. It can be explained that higher amounts of adsorbents would spend longer time to settle down, make it harder to collect from the aqueous solutions [42]. In conclusion, $7 \mathrm{mg}$ of magnetic MOF-5 composites were the minimum amount of adsorbents to attain the best extraction efficiency. Thus, $7 \mathrm{mg}$ was employed as the amount of adsorbent for further studies.

\subsubsection{Effect of sample solution $\mathrm{pH}$}

The $\mathrm{pH}$ value of the sample solution is very significant for the adsorption efficiency. The $\mathrm{pH}$ value of the sample solution would influence both the form of four pesticides and the surface binding sites of magnetic MOF-5. The effect of $\mathrm{pH}$ was investigated by varying the $\mathrm{pH}$ from 2.0 to 7.0. As presented in Fig. 3B, the extraction efficiencies of carbendazim, triadimefon and fenpyroximate increased within $\mathrm{pH}$ 2.0-6.0 followed by decreasing when $\mathrm{pH}>6.0$. The extraction efficiencies of chlorfenapyr increased within $\mathrm{pH}$ 2.0-3.0 then decreased a bit at $\mathrm{pH} 4.0$; interestingly, the highest extraction efficiency was also acquired at $\mathrm{pH}$ 6.0. For explanation of the effect of $\mathrm{pH}$, the zeta potential change of magnetic MOF-5 was measured at different solution $\mathrm{pH}$, as shown in Fig. S8a, and the related zeta potential change of $\mathrm{NH}_{2}-\mathrm{Fe}_{3} \mathrm{O}_{4}$ was shown in Fig. S8b. The isoelectric point of magnetic MOF-5 was attained at about 6.0, which means at pH 6.0 the magnetic MOF-5 was hydrophobic. From the $\log K_{\text {ow }}$ data of four pesticides (shown in Fig. 1), we can see that three pesticides (triadimefon, fenpyroximateand chlorfenapyr) are strong hydrophobic $\left(\log \mathrm{K}_{\mathrm{ow}}>3\right)$. Therefore, at $\mathrm{pH} 6.0$, the interaction between magnetic MOF-5 and three pesticides (triadimefon, fenpyroximateand chlorfenapyr) was mainly hydrophobic interactions. In addition, the benzene rings within magnetic MOF-5 and pesticides would induce $\pi-\pi$ interaction. As for carbendazim, the $\log \mathrm{K}_{\mathrm{ow}}$ of carbendazim is 1.51 (in the range of $0.5-3$ ), which means that carbendazim is middle hydrophobic. The $p \mathrm{~K}_{\mathrm{a}}$ of carbendazim is about 5.0. So at pH 6.0 the carbendazim molecules were neutral and anionic species. The part of neutral carbendazim molecules might interact with magnetic MOF-5 by hydrophobic interaction and $\pi-\pi$ interaction. At other $\mathrm{pH}$ values, the magnetic MOF-5 composites were positively or negatively charged, but tri-
Table 2

Intra-day and inter-day precision (RSDs,\%) for the magnetic MOF-5 based MSPE coupled with HPLC for the determination of heterocyclicpesticides.

\begin{tabular}{llll}
\hline Heterocyclic pesticides & Spiked $(\mu \mathrm{g} / \mathrm{L})$ & Inter-day $(n=6)$ & Intra-day $(n=6)$ \\
\hline Carbendazim & 5 & 6.68 & 4.23 \\
& 50 & 5.9 & 5.91 \\
Triadimefon & 100 & 5.65 & 6.32 \\
& 5 & 6.53 & 7.12 \\
Chlorfenapyr & 50 & 3.37 & 5.71 \\
& 100 & 4.08 & 4.16 \\
& 5 & 3.71 & 6.23 \\
Fenpyroximate & 50 & 4.13 & 3.31 \\
& 100 & 7.11 & 4.46 \\
& 5 & 2.98 & 6.52 \\
& 50 & 6.06 & 3.46 \\
& 100 & 3.52 & 5.53 \\
\hline
\end{tabular}

adimefon, fenpyroximate and chlorfenapyr were neutral forms. So the pesticide molecules cannot interact with magnetic MOF-5, and the extraction efficiencies were lower than that at $\mathrm{pH}$ 6.0. When $\mathrm{pH}$ was much less than 5.0, carbendazim and magnetic MOF-5 was all positively charged, so magnetic MOF-5 and carbendazim were repulsive. When $\mathrm{pH}$ was higher than 6.0, the magnetic MOF5 and carbendazim were all negatively charged, so they also were repulsive. The electrostatic repulsion between pesticide and magnetic MOF-5 led to lower extraction efficiencies. Therefore, sample solutions were analyzed at pH 6.0 in the following procedures.

\subsubsection{Effect of extraction time}

The adsorption efficiencies at different extraction time (3-30 min) were studied. Fig. 3C shows that the extraction efficiencies for four pesticides reached their maxima at $12 \mathrm{~min}$ and then remained almost constant, indicating that 12 min was sufficient for the adsorption process to achieve equilibrium. Therefore, 12 min was selected as the optimum extraction time.

\subsubsection{Effect of desorption condition}

The properties of different desorption solvents would influence the extraction efficiency of MSPE process. Four organic solvents, i.e., methanol, acetonitrile, acetone and ethyl acetate were examined as desorption solvents. Fig. 3D depicts that the best desorption capabilities towards the four pesticides were provided by ethyl acetate, which can better disrupt interactions between magnetic MOF-5 and pesticides. Therefore, ethyl acetate was chosen as the desorption solvent for further experiments.

Then, the effects of different volumes of ethyl acetate and desorption time on the extraction efficiencies were investigated. As seen from Fig. $3 \mathrm{E}, 2 \times 2 \mathrm{~mL}$ of ethyl acetate was not enough to elute all the pesticides from the magnetic MOF-5, while $2 \times 4 \mathrm{~mL}$ ethyl acetate didn't provide any significant change on extraction efficiency. So in the further experiment, $2 \times 3 \mathrm{~mL}$ of ethyl acetate eluted was adopted. Different desorption time of 4,6 , and 8 min were evaluated and Fig. $3 \mathrm{~F}$ shows that 6 min was enough to accomplish one desorption period. Therefore, the desorption time was set at $6 \mathrm{~min}$.

\subsubsection{Effect of salt concentration}

Salt concentration in water samples plays an important role in extraction efficiency. The ionic strength and viscosity of water solutions can be changed by salt addition, which would affect the interactions between the sorbent and analytes. Thus, the effect of salinity on the extraction of pesticides was investigated by adding various concentration of $\mathrm{NaCl}$ from 0 to $10.0 \%(\mathrm{w} / \mathrm{v})$. As shown in Fig. $3 F$, the extraction efficiencies for the four pesticides increased along with the concentration of $\mathrm{NaCl}$ increasing from 0 to $10.0 \%$. This phenomenon can be explained that at higher ionic strength, 

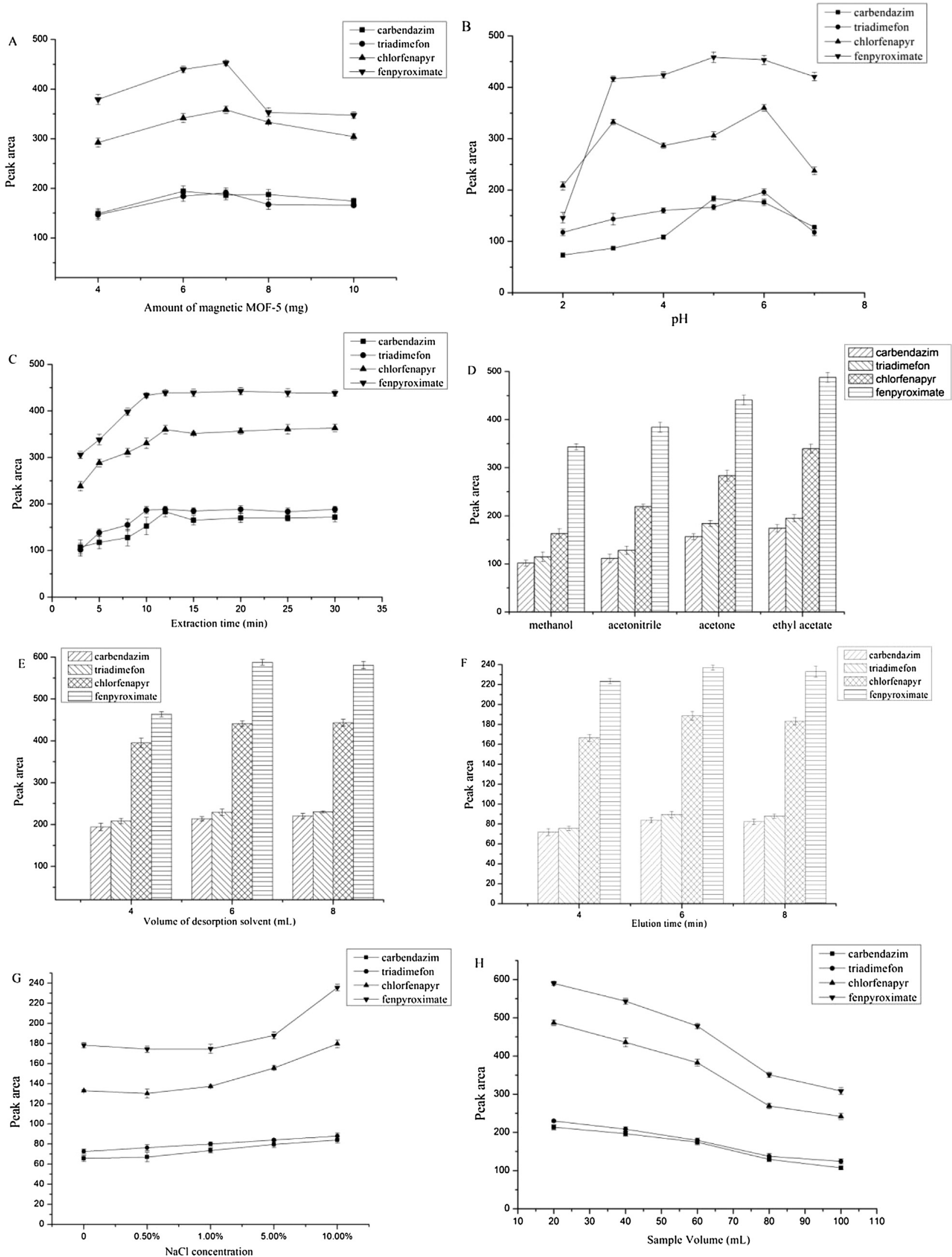

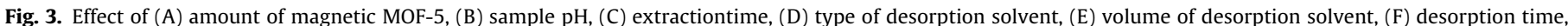

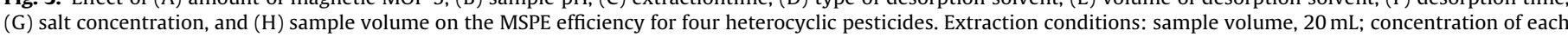

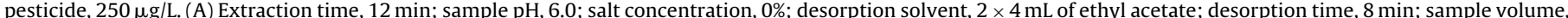

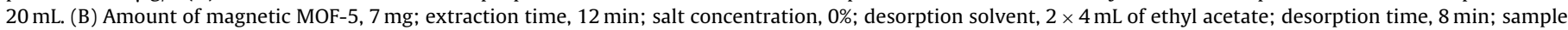

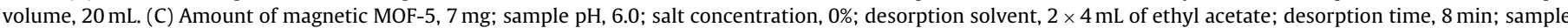

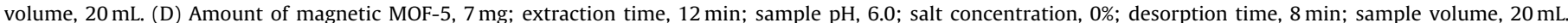



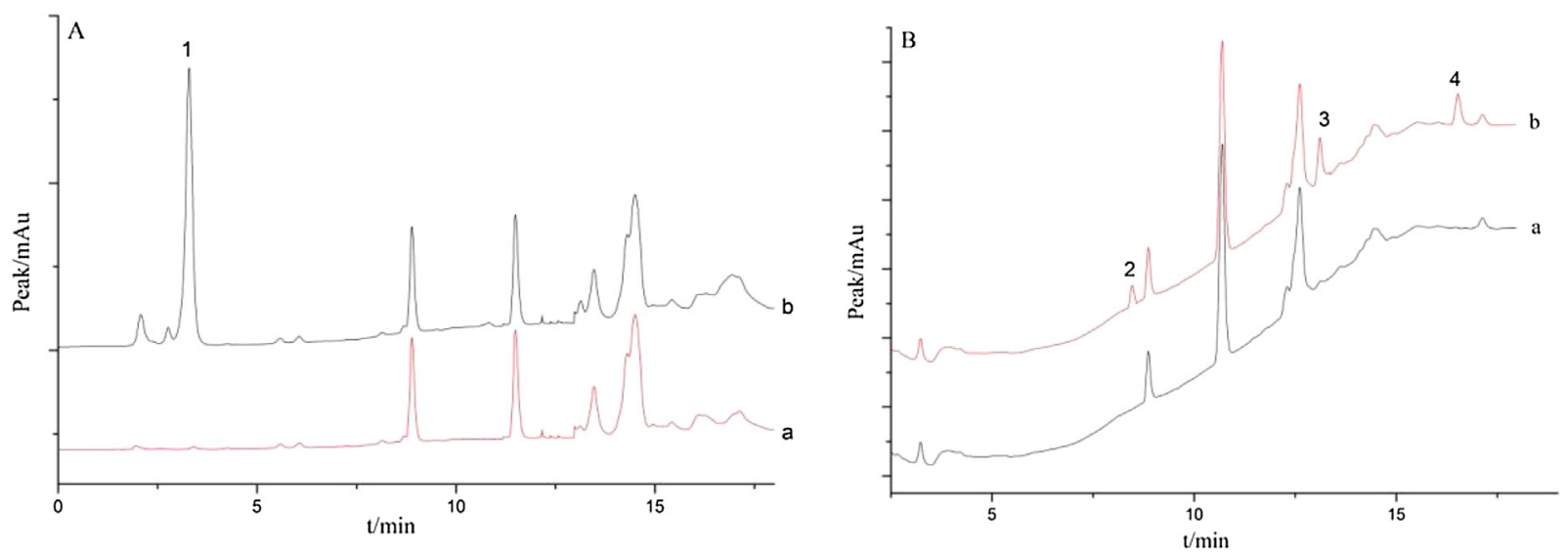

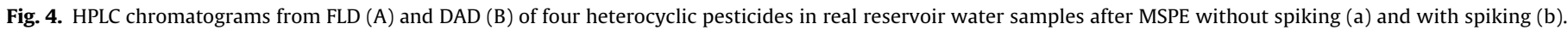
Peak identification: FLD for (1) carbendazim; DAD for (2) triadimefon, (3) chlorfenapyr, and (4) fenpyroximate. Extraction conditions were the optimal conditions.

Table 3

Determination of four heterocyclic pesticides and method recoveries in real water samples.

\begin{tabular}{|c|c|c|c|c|c|c|c|}
\hline \multirow[t]{2}{*}{ Heterocyclic pesticides } & \multirow{2}{*}{$\begin{array}{l}\text { Spiked } \\
(\mu \mathrm{g} / \mathrm{L})\end{array}$} & \multicolumn{2}{|c|}{ NC reservoirwater ${ }^{\mathrm{a}}$} & \multicolumn{2}{|c|}{ QH reservoirwater ${ }^{\mathrm{b}}$} & \multicolumn{2}{|l|}{ River water $^{\mathrm{c}}$} \\
\hline & & Found $(\mu \mathrm{g} / \mathrm{L})$ & Recovery $(\% \pm \mathrm{RSD}, \mathrm{n}=3)$ & Found $(\mu \mathrm{g} / \mathrm{L})$ & Recovery $(\% \pm \mathrm{RSD}, \mathrm{n}=3$ ) & Found $(\mu \mathrm{g} / \mathrm{L})$ & Recovery $(\% \pm \mathrm{RSD}, \mathrm{n}=3$ ) \\
\hline \multirow{4}{*}{ Carbendazim } & 0 & $\mathrm{ND}^{\mathrm{d}}$ & & ND & & ND & \\
\hline & 5.0 & 5.42 & $108.33 \pm 7.0$ & 4.89 & $97.74 \pm 5.39$ & 5.33 & $106.67 \pm 4.42$ \\
\hline & 50.0 & 43.95 & $87.89 \pm 6.53$ & 43.17 & $86.34 \pm 6.92$ & 43.24 & $86.47 \pm 5.64$ \\
\hline & 100.0 & 93.17 & $93.17 \pm 4.20$ & 95.30 & $95.30 \pm 4.29$ & 96.41 & $96.41 \pm 3.67$ \\
\hline \multirow[t]{4}{*}{ Triadimefon } & 0 & ND & & ND & & ND & \\
\hline & 5.0 & 4.84 & $96.76 \pm 6.92$ & 4.44 & $88.76 \pm 6.29$ & 4.41 & $88.19 \pm 6.12$ \\
\hline & 50.0 & 46.16 & $92.32 \pm 4.60$ & 45.51 & $91.02 \pm 4.60$ & 47.14 & $94.28 \pm 5.00$ \\
\hline & 100.0 & 89.28 & $89.28 \pm 3.95$ & 91.16 & $91.16 \pm 3.95$ & 93.14 & $93.14 \pm 2.96$ \\
\hline \multirow[t]{4}{*}{ Chlorfenapyr } & 0 & ND & & ND & & ND & \\
\hline & 5.0 & 4.26 & $85.15 \pm 6.26$ & 4.39 & $87.80 \pm 7.38$ & 4.02 & $80.30 \pm 4.40$ \\
\hline & 50.0 & 45.38 & $90.76 \pm 7.41$ & 47.87 & $95.75 \pm 6.72$ & 47.20 & $94.41 \pm 4.19$ \\
\hline & 100.0 & 94.46 & $94.46 \pm 5.28$ & 102.92 & $102.92 \pm 5.89$ & 97.40 & $97.40 \pm 4.37$ \\
\hline \multirow[t]{4}{*}{ Fenpyroximate } & 0 & ND & & ND & & ND & \\
\hline & 5.0 & 4.05 & $81.09 \pm 6.46$ & 4.34 & $86.73 \pm 7.79$ & 4.33 & $86.61 \pm 7.94$ \\
\hline & 50.0 & 50.46 & $100.92 \pm 6.30$ & 47.50 & $94.99 \pm 7.22$ & 47.85 & $95.70 \pm 5.11$ \\
\hline & 100.0 & 82.03 & $82.03 \pm 3.18$ & 86.19 & $86.19 \pm 6.81$ & 90.86 & $90.86 \pm 3.86$ \\
\hline
\end{tabular}

a From NuochengReservoir.

b FromQianhan Reservoir.

c From Yellow River.

d Not detected.

dissociative $\mathrm{NaCl}$ surrounding the adsorption sites on the surface of magnetic MOF-5 would neutralize the surface charge of the adsorbent, and thus enhance the hydrophobic force between magnetic MOF-5 and pesticide molecules [43]. However, too high salt concentration would impede the elution process by making the desorption solvent viscous. Therefore, the concentration of $\mathrm{NaCl}$ was adjusted to $10 \%$ in the following extractions.

\subsubsection{Effect of sample volume}

The sample volume can alter the preconcentration factor, which directly affects the sensitivity of this method. Therefore, the effect of sample volume on extraction efficiency was investigated. For this purpose, $7 \mathrm{mg}$ of magnetic MOF-5composites were suspended in different sample volumes $(20,40,60,80$ and $100 \mathrm{~mL})$ with the fixed amount of analytes. Fig. $3 \mathrm{H}$ shows that the peak areas were decreased when sample volume increased from 20 to $80 \mathrm{~mL}$. The sample volume could impact the mass distribution equilibrium
[44], thus, it was harder for larger sample volume to achieve mass transfer equilibrium from liquid to solid phase. The peak areas were sharply decreased as sample volume increased from 60 to $80 \mathrm{~mL}$. Therefore, as a compromise between the extraction efficiency and preconcentration factor, $60 \mathrm{~mL}$ was employed as the sample volume.

\subsection{Analytical performance of MSPE-HPLC}

Under optimum conditions described above, analysis performance of the magnetic MOF-5 adsorbents based MSPE coupled with HPLC towards four pesticides was assessed. Stock solutions were serially diluted with pure water to obtain four standard solutions at seven different concentration levels (1.0, 5.0, 50.0, 100.0, $200.0,250.0$, and $500.0 \mu \mathrm{g} / \mathrm{L}$ for individual pesticide).Four calibration curves were established within the investigated concentration range, respectively. As listed in Table 1 , excellent linearity was

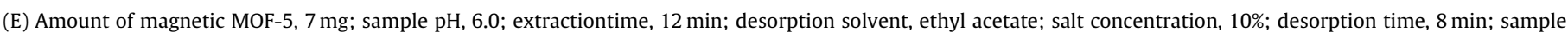

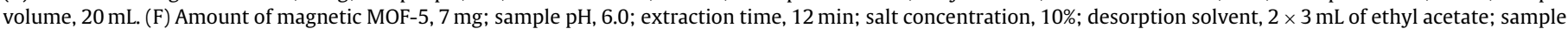

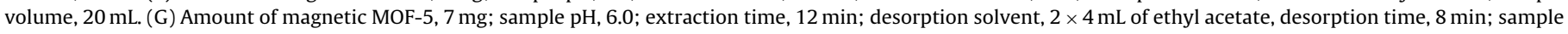
volume, $20 \mathrm{~mL}$. (H) Amount of magnetic MOF-5, $7 \mathrm{mg}$; sample $\mathrm{pH}, 6.0$; extraction time, $12 \mathrm{~min}$; salt concentration, $10 \%$; desorption solvent, $2 \times 3 \mathrm{~mL}$ of ethyl acetate. 
Table 4

Method performance comparison for heterocyclicpesticides in water samples.

\begin{tabular}{|c|c|c|c|c|}
\hline Heterocyclic pesticides & Pretreatment techniques & Detection techniques & LODs $(\mu \mathrm{g} / \mathrm{L})$ & Ref. \\
\hline Ametryn, atrazine, diuron and fipronil & C18-SPE & HPLC-DAD & $20-50$ & {$[10]$} \\
\hline Fipronil, chlorfenapyr, fenpyroximate, flusilazole & MWCNTs-SPE & HPLC-DAD & $0.008-0.019$ & {$[14]$} \\
\hline clofentezine,chlorfenapyr & IL-MSPE & HPLC-DAD & $0.4-0.5$ & [39] \\
\hline Fipronil, chlorfenapyr, fenpyroximate, flusilazole & $\mathrm{Fe}_{3} \mathrm{O}_{4} @ \mathrm{SiO}_{2}-\mathrm{MIL}-101-\mathrm{MSPE}$ & HPLC-DAD & $0.3-1.5$ & [34] \\
\hline Carbendazim, triadimefon, chlorfenapyr, fenpyroximate & Magnetic MOF-5-MSPE & HPLC-DAD/FLD & $0.4-0.11$ & This work \\
\hline
\end{tabular}

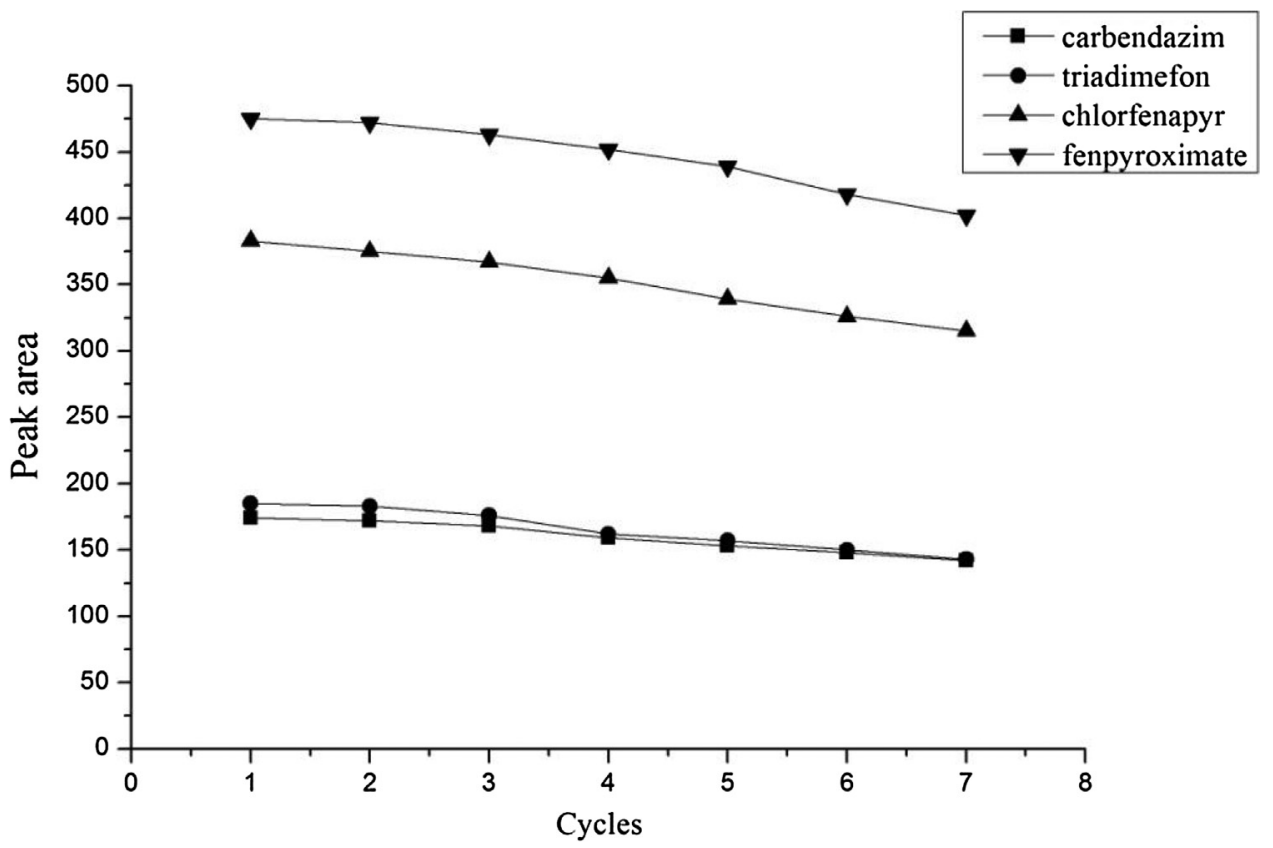

Fig. 5. Reusability of the magnetic MOF-5 composites for MSPE.

obtainedwithin $0.3-500.0 \mu \mathrm{g} / \mathrm{L}$ for carbendazim and triadimefon, and $0.1-500.0 \mu \mathrm{g} / \mathrm{L}$ for chlorfenapyr and fenpyroximate, with correlation coefficients $(r)$ between 0.9992 and 0.9997 . The limits of detection (LODs) calculated at a signal to noise ratio of $3(\mathrm{~S} / \mathrm{N}=3)$ were attained from 0.04 to $0.11 \mu \mathrm{g} / \mathrm{L}$ (Table 1). Moreover, the limits of quantitation (LOQs) were evaluated on the basis of $\mathrm{S} / \mathrm{N}=10$ and ranged from 0.13 to $0.35 \mu \mathrm{g} / \mathrm{L}$ (Table 1 ). The LODs for all the four pesticides are lower than maximum residue level (MRL) of $1 \mu \mathrm{g} / \mathrm{L}$ in European Union surface water intended for the abstraction of drinking water regulations (75/440/EEC) [45]. Thus, this developed method could meet the requirements of MRLs detection of the four pesticides in surface water samples. On the other hand, the intra-day and inter-day precisions were used to investigate the reproducibility of the method. Each spiked concentration level was repeatedly performed six times in one day and in six consecutive days to evaluate the intra-day and the inter-day variations, respectively. As shown in Table 2, the calculated relative standard deviations (RSDs) for intra-day recoveries were below $7.12 \%$ and for inter-day were less than $7.11 \%$. These results indicated the magnetic MOF-5 were excellent adsorbents for the MSPE-HPLC method, which could highly sensitive and accurate enrichment and determination of the four heterocyclic pesticides at trace levels simultaneously.

\subsection{Application of the MSPE-HPLC to real environmental water samples}

The developed MSPE-HPLC method was further applied to three water samples from Qianhan Reservoir, Nuo cheng Reservoir and the Yellow River. Representative HPLC chromatograms of reser- voir water samples were displayed in Fig. 4 (A for FLD signal, B for DAD signal). The chromatograms in Fig. $4 \mathrm{~B}$ were obtained from the DAD signal, which was more likely to be affected by the change of mobile phase, thus the baseline of the chromatogram shifted seriously. However, the chromatograms in Fig. 4A were obtained from the FLD signal, so the change of mobile phase did not affect the signal of the baseline. As seen, there were no endogenous target pesticides detected in the real water samples after MSPE (curve a of Fig. 4 and Table 3), and the peaks of four pesticides appeared after spiking (curve b of Fig. 4). As listed in Table 3, the recoveries of four pesticides at three spiked concentrations were in a range of $81.09-108.33 \%$ with RSDs of $3.18-7.41 \%$ for Nuocheng reservoir water sample, 86.19-102.92\% with RSDs of 3.95-7.79\% for Qianhan reservoir water sample and $80.30-106.67 \%$ with RSDs of 2.96-7.94\% for Yellow river water sample. Thus, the established MSPE-HPLC method was practically available for the determination of trace heterocyclic pesticides in complex water samples.

\subsection{Regeneration of magnetic MOF-5 composites for MSPE}

Reusability indicates the efficiency and cost effectiveness of an adsorbent which is an important parameter to practical and commercial applications. To investigate the possibility of reusing the magnetic MOF-5, several adsorption-desorption cycles for MSPE were carried out. As seen, Fig. 5 indicates that the reusability efficiency of the magnetic MOF- 5 was still high after seven successive cycles. The results demonstrated that the magnetic MOF-5 possessed high stability and good reusability, and could be readily regenerated, which is highly desirable for MSPE. 


\subsection{Method performance comparison}

Method performance of the developed MSPE-HPLC was compared with other reported for the heterocyclic pesticides. As shown in Table 4, several pretreatment methods such as, SPE [10,14], MSPE $[46,36]$ were presented. Compared to the same HPLC-DAD determination with different pretreatment techniques [10,14,36,46], our method achieves lower LODs. The method using MWCNTs as SPE adsorbents combined with HPLC shows the lowest LODs [14]. However, the conventional SPE process needs to spend nearly $4 \mathrm{~h}$, while the dispersed MSPE material could be rapidly collected from the water samples and the pretreatment procedure could be completed in only $20 \mathrm{~min}$. Therefore, the developed MSPE-HPLC method has been demonstrated to be simple, sensitive, cost-effective and potentially applicable for the determination of four heterocyclic pesticides from environmental water samples.

\section{Conclusions}

In this work, magnetic MOF-5 composites were prepared and successfully applied as the MSPE adsorbents towards four heterocyclic pesticides in environmental water samples along with HPLC-DAD/FLD. MOFs materials enabled high adsorption capability and magnetic properties simplified operation procedure; the combined MOFs-MSPE enabled rapidity, convenience, good reliability and high sensitivity. Furthermore, the selectivity of magnetic MOFs should be enhanced by utilizing functional groups, specific interactions/combinations, and thereby provide promising perspectives for more targeted pollutants in more complicated matrices.

\section{Conflict of interest}

The authors declare no conflict of interest.

\section{Acknowledgments}

The authors acknowledge support from the National Natural Science Foundation of China $(21547002,21477160)$.

\section{Appendix A. Supplementary data}

Supplementary data associated with this article can be found, in the online version, at https://doi.org/10.1016/j.chroma.2018.04. 034.

\section{References}

[1] M.A.J. Huijbregts, L.J.A. Rombouts, A.M.J. Ragas, D. van de Meent, Human-toxicological effect and damage factors of carcinogenic and noncarcinogenic chemicals for life cycle impact assessment, Integr. Environ. Assess. Manag. 1 (2005) 181-192.

[2] G.J.M. Hans, A.A. Rooseboom, R. van Dam, M. Roding, K. Arondeus, S. Sunarto, Modification and revalidation of the ethyl acetate-based multi-residue method for pesticides in produce, Anal. Bioanal. Chem. 389 (2007) 1715-1754.

[3] V.A. Belmonte, F.A. Garrido, V.J.L. Martinez, Monitoring of pesticides in agricultural water and soil samples from Andalusia by liquid chromatography coupled to mass spectrometry, Anal. Chim. Acta 538 (2005) 117-127.

[4] T. Wang, J. Hu, C. Liu, Simultaneous determination of insecticide fipronil and its metabolites in maize and soil by gas chromatography with electron capture detection, Environ. Monit. Assess. 186 (2014) 2767-2774.

[5] J.J. Jimenez, J.L. Bernal, M.J. Del Nozal, M.T. Martín, R. Mayo, Sample preparation methods to analyze fipronil in honey by gas chromatography with electron-capture and mass spectrometric detection, J. Chromatogr. A 1187 (2008) 40-45

[6] Y.S. Cao, J.X. Chen, Y.L. Wang, J. Liang, L.H. Chen, Y.T. Lu, HPLC/UV analysis of chlorfenapyr residues in cabbage and soil to study the dynamics of different formulations, Sci. Total Environ. 350 (2005) 38-46.

[7] Y. Liu, E.C. Zhao, W.T. Zhu, H.X. Gao, Z.Q. Zhou, Determination of four heterocyclic insecticides by ionic liquid dispersive liquid-liquid microextraction in water samples, J. Chromatogr. A 1216 (2009) 885-891.
[8] D.B. Tomasini, M.R.F. Sampaio, S.S. Caldas, J.G. Buffon, F.B.A. Duarte, E.G. Primel, Simultaneous determination of pesticides and 5-hydroxymethylfurfural in honey by the modified QuEChERS method and liquid chromatography coupled to tandem mass spectrometry, Talanta 99 (2012) 380-386.

[9] M.Z. Lacroix, S. Puel, P.L. Toutain, C. Viguié, Quantification of fipronil and its metabolite fipronilsulfone in rat plasma over a wide range of concentrations by LC/UV/MS, J. Chromatogr. B 878 (2010) 1934-1938.

[10] L.T.D. Cappelinia, D. Cordeiroa, S.H.G. Brondib, K.R. Prietoa, E.M. Vieira, Development of methodology for determination of pesticides residue in water bySPE/HPLC/DAD, Environ. Technol. 33 (2012) 2299-2304.

[11] J.L. Vuílchez, A. Prieto, L. Araujo, A. Navalón, Determination of fipronil by solid phase microextraction and gas chromatography-mass spectrometry, J. Chromatogr. A 919 (2001) 215-221.

[12] N. Pourreza, S. Rastegarzadeh, A. Larki, Determination of fungicide carbendazim in water and soil samples using dispersive liquid-liquid microextraction and microvolume UV-vis spectrophotometry, Talanta 134 (2015) 24-29.

[13] Y. Liu, E. Zhao, W.T. Zhu, H.X. Gao, Z.Q. Zhou, Determination of four heterocyclic insecticides by ionic liquid dispersive liquid-liquid microextraction in water samples, J. Chromatogr. A 1216 (2009) 885-891.

[14] J.P. Ma, X. Lu, Y. Xia, F.L. Yan, Determination of pyrazole and pyrrole pesticides in environmental water samples by solid-phase extraction using multi-walled carbon nanotubes as adsorbent coupled with high-performance liquid chromatography, J. Chromatogr. Sci. 53 (2015) 380-384.

[15] J.H. Li, R.C. Dong, X.Y. Wang, H. Xiong, S.F. Xu, D.Z. Shen, X.L. Song, L.X. Chen, One-pot synthesis of magnetic molecularly imprinted microspheres by RAFT precipitation polymerization for the fast and selective removal of17ß-estradiol, RSC Adv. 5 (2015) 10611-10618.

[16] J.P. Ma, L.H. Jiang, G.G. Wu, Y. Xia, W.H. Lu, J.H. Li, L.X. Chen, Determination of six sulfonylurea herbicides in environmental water samples by magnetic solid-phase extraction using multi-walled carbon nanotubes as adsorbents coupled with high-performance liquid chromatography, J. Chromatogr. A 1466 (2016) 12-20.

[17] N.L. Rosi, J. Eckert, M. Eddaoudi, D.T. Vodak, J. Kim, M. O’keeffe, O.M. Yaghi, Hydrogen storage in microporous metal-organic frameworks, Science 300 (2003) 1127-1129.

[18] D.N. Dybtsev, H. Chun, S.H. Yoon, D. Kim, K. Kim, Microporous manganese formate: a simple metal-organic porous material with high framework stability and highly selective gas sorption properties, J. Am. Chem. Soc. 126 (2004) 32-33.

[19] L. Wang, Y. Han, X. Feng, J. Zhou, P. Qi, B. Wang, Metal-organic frameworks for energy storage: batteries and super capacitors, Coord. Chem. Rev. 307 (2015) 361-381.

[20] P. Horcajada, C. Serre, M. Vallet-Regi, M. Sebban, F. Taulelle, G. Férey, Metal-organic frameworks as efficient materials for drug delivery, Angew. Chem. Int. Ed. 45 (2006) 5974-5978.

[21] P. Horcajada, T. Chalati, C. Serre, B. Gillet, C. Sebrie, T. Baati, J.F. Eubank, D. Heurtaux, P. Clayette, C. Kreuz, J.S. Chang, Y.K. Hwang, V. Marsaud, P.N. Bories, L. Cynober, S. Gil, G. Ferey, P. Couvreur, R. Gref, Porousmetal-organic-framework nanoscale carriers as a potential platform for drug delivery and imaging, Nat. Mater. 9 (2010) 172-178.

[22] S. Rostamnia, H. Alamgholiloo, X. Liu, Pd-grafted open metal site copperbenzene-1,4-dicarboxylate metal organic frameworks (Cu-BDC MOF's) as promising interfacial catalysts for sustainable Suzuki coupling, J. Colloid Interface Sci. 469 (2016) 310-317.

[23] J. Lee, O.K. Farha, J. Roberts, K.A. Scheidt, S.T. Nguyen, J.T. Hupp, Metal-organic framework materials as catalysts, Chem. Soc. Rev. 38 (2009) 1450-1459.

[24] X. Wang, N.S. Ye, Recent advances in metal-organic frameworks and covalent organic frameworks for sample preparation and chromatographic analysis, Electrophoresis 38 (2017) 3059-3078.

[25] Y.H. Wang, M. Rui, G.H. Lu, Recent applications of metal-organic frameworks in sample pretreatment, J. Sep. Sci. 41 (2018) 180-194.

[26] Y.Y.Zhou, X.P. Yan, K.N. Kim, S.W. Wang, M.G. Liu, Exploration of coordination polymer as sorbent for flow injection solid-phase extraction on-line coupled with high-performance liquid chromatography for determination of polycyclic aromatichydrocarbons in environmental materials, J. Chromatogr. A 1116 (2006) 172-178.

[27] X.P. Dai, X.N. Jia, P. Zhao, T. Wang, J. Wang, P.T. Huang, L. He, X.H. Hou, A combined experimental/computational study on metal-organic framework MIL-101(Cr) as a SPE sorbent for the determination of sulphonamides in environmental water samples coupling with UPLC-MS/MS, Talanta 154 (2016) 581-588.

[28] Z.Z. Huang, H.K. Lee, Micro-solid-phase extraction of organochlorine pesticides using porousmetal-organic framework MIL-101 as sorbent, J. Chromatogr. A 1401 (2015) 9-16.

[29] X.Y. Cui, Z.Y. Gu, D.Q. Jiang, Y. Li, H.F. Wang, X.P. Yan, In Situ hydrothermal growth of metal-organic framework 199 films on stainless steel fibers for solid-phase microextraction of gaseous benzene homologues, Anal. Chem. 81 (2009) 9771-9777.

[30] C.T. He, J.Y. Tian, S.Y. Liu, G.F. Ouyang, J.P. Zhang, X.M. Chen, A porous coordination framework for highly sensitive and selective solid-phase microextraction of non-polar volatile organic compounds, Chem. Sci. 4 (2013) $351-356$.

[31] N.C. Burtch, H. Jasuja, K.S. Walton, Water stability and adsorption in metal organic frameworks, Chem. Rev. 114 (2014) 10575-10612. 
[32] T.M. De, R. Jonchiere, P. Pullumbi, F.X. Coudert, A.H. Fuchs, How can a hydrophobic MOF be water-unstable? Insight into the hydration mechanism of IRMOFs, Chem. Phys. Chem. 13 (2012) 3497-3503.

[33] S.H. Huo, X.P. Yan, Facile magnetization of metal-organic framework MIL-101 for magnetic solid-phase extraction of polycyclic aromatic hydrocarbons in environmental water samples, Analyst 137 (2012) 3445-3451.

[34] J.P. Ma, Z.D. Yao, L.W. Hou, W.H. Lu, Q.P. Yang, J.H. Li, L.X. Chen, Metal organic frameworks (MOFs) for magnetic solid-phase extraction of pyrazole/pyrrole pesticides in environmental water samples followed by HPLC-DAD determination, Talanta 161 (2016) 686-692.

[35] A. Bagheri, M. Taghizadeh, M. Behbahani, A.A. Asgharinezhad, M. Salarian, A. Dehghani, H. Ebrahimzadeh, M.M. Amini, Synthesis and characterization of magnetic metal-organic framework (MOF) as a novel sorbent, and its optimization by experimental design methodology for determination of palladium in environmental samples, Talanta 99 (2012) 132-139.

[36] X.M. Liu, Z.W. Sun, G.N. Chen, W.W. Zhang, Y.P. Cai, R.M. Kong, X.Y. Wang, Y.R. Suo, J.M. You, Determination of phthalate esters in environmental water by magnetic Zeolitic Imidazolate Framework-8 solid-phase extraction coupled with high-performance liquid chromatography, J. Chromatogr. A 1049 (2015) 46-52.

[37] Z.X. Lv, Z.W. Sun, C.H. Song S.M. Lu, G. Chen, J.M. You, Sensitive and background-free determination of thiols from wastewater samples by MOF-5 extraction coupled with high-performance liquid chromatography with fluorescence detection using a novel fluorescence probe of carbazole-9-ethyl-2-maleimide, Talanta 161 (2016) 228-237.

[38] S. Bahrani, M. Ghaedi, K. Dashtian, A. Ostovan, M.J.K. Mansoorkhanic, A. Salehid, MOF-5(Zn)-Fe2O4 nanocomposite based magnetic solid-phase microextraction followed by HPLC-UV for efficient enrichment of colchicines in root of colchicium extracts and plasma samples, J. Chromatogr. B 1067 (2017) 45-52.
[39] Y.L. Hu, Z.L. Huang, J. Liao, Chemical bonding approach for fabrication of hybrid magnetic metal-organic framework-5: high efficient adsorbents for magnetic enrichment of trace analyte, Anal. Chem. 85 (2013) 6885-6893.

[40] Z. Zhang, L.X. Chen, F.F. Yang, J.H. Li, Uniform core-shell molecularly imprinted polymers: a correlation study between shell thickness and binding capacity, RSC Adv. 4 (2014) 31507-31514.

[41] X.D. Xin, Q. Wei, J. Yang, L.G. Yan, R. Feng, G.D. Chen, B. Du, H. Li, Highly efficient removal of heavy metal ions by amine-functionalized mesoporous $\mathrm{Fe}_{3} \mathrm{O}_{4}$ nanoparticles, Chem. Eng. J. 184 (2012) 132-140.

[42] P.R. Bautista, C.M. Benito, V. Pino, J. Pasán, J.H. Ayala, C.R. Pérez, A.M. Afonso, The metal-organic framework HKUST-1 as efficient sorbent in a vortex-assisted dispersive micro solid-phase extraction of parabens from environmental waters, cosmetic creams, and human urine, Talanta 139 (2015) 13-20.

[43] M. Campinas, M.J. Rosa, The ionic strength effect on microcystin and natural organic matter surrogate adsorption onto PAC, J. Colloid Interface Sci. 299 (2006) 520-529.

[44] G.J. Zhang, X.H. Zang, Z. Li, C. Wang, Z. Wang, Polydimethylsiloxane/metal-organic frameworks coated fiber for solid-phase microextraction of polycyclic aromatic hydrocarbons in river and lake water samples, Talanta 129 (2014) 600-605.

[45] 75/440/EEC. Concerning the quality required of surface water intended for the abstraction of drinking water in the Member States (EU).

[46] B. Peng, J.H. Zhang, R.H. Lu, S.B. Zhang, W.F. Zhou, H.X. Gao, Dispersive micro-solid phase extraction based on self assembling, ionic liquid-coated magnetic particles for the determination of clofentezine and chlorfenapyr in environmental water samples, Analyst 138 (2013) 6834-6843. 